\title{
Invariant Features Extraction for Banknote Classification
}

\author{
Peng Wang ${ }^{1} \quad$ Peng Liu ${ }^{2}$ \\ ${ }^{1}$ Electronics and Information College, Heilongjiang Institute of Science and Technology, \\ Harbin 150027, China \\ ${ }^{2}$ College of Computer Science and Technology, Harbin Institute of Technology, Harbin \\ 150001, China
}

\begin{abstract}
An invariant feature extraction method is proposed for banknote classification. The movement of banknote is complex in the channel of financial instruments. The scale is various. The rotation and translation are also to occur. The method of feature extraction is insensitive to the variety of scale, rotation and translation. It decreases the data variety and improves the reliability of banknote classification. Furthermore, the computation complexity is low in order to meet to the requirement of real-time banknote image processing and classification. The invariant feature extraction method has performed very well when they are applied in banknote sorters.
\end{abstract}

Keywords: banknote classification; invariant feature; neural networks; image processing; hardware plane

\section{1 introduction}

Supervisory classifiers, such as neural networks, have been effectively used for banknote classification in many financial instruments ${ }^{[1][2]}$. Although these classifiers are robust in recognition of different types of bills, such as dirty bills, very old ones, misclassification is unavoidable. In financial instruments,

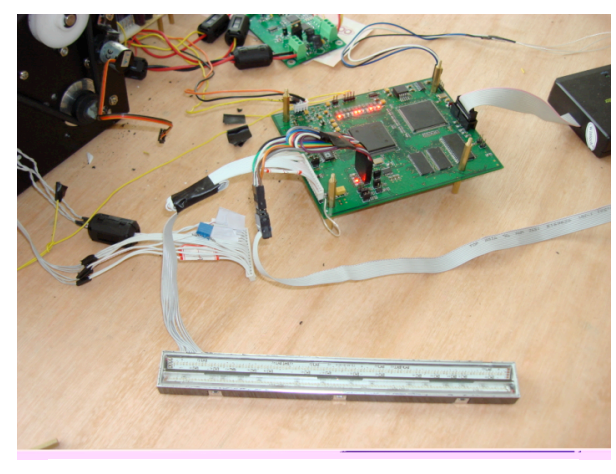

Figure 1 Hardware plane and CIS

misclassification will bring tremendous loss. A well-trained classifier can result in misclassification. It is mainly due to the large variety and distortion of training samples. In real applications, the speed that a banknote passes the image sensor is very fast. The process of imaging is complex. Because of requirement of real-time, the resolution of image is very

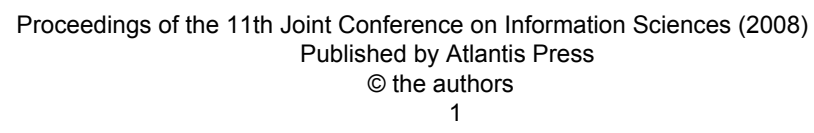


low. Therefore, invariant features extraction to decrease the data variety in real-time is one of crucial problem.

A.Ahmadi proposed local PCA $^{[3]}$ (Principal Components Analysis) to improve the reliability. This method overcomes the limitation of PCA, which is global linearity. The improvement of reliability is clear, but it can't satisfy the requirement of real-time because many matrix multiplication results to high computation complexity. A fuzzy clustering method was proposed in Reference $^{[4]}$. The multi-spectrum images were used to classify the banknotes. Because the fuzzy clustering classifier utilized the more information, its accuracy is reliable. However, the more hardware resource is used to store the multi-spectrum images. It is uneasy to be implemented in embedded system.

This paper shows an effective method to classify banknote. First, a rotation, translation and scale invariant feature extraction method is proposed. This feature extraction method is suit for the low quality images which are acquired at high speed banknote channel. Furthermore, the computation is sample in order to finish the banknote image processing and classification in real-time. Then, a 3-layer BP neural networks is used predict the face and value of a banknote. The feature extraction method has performed very well when they are applied in banknote sorters.

The organization of this paper is as follows: the hardware plane is described briefly in Section 2, and the invariant feature extraction method is presented in Section 3. Section 4 deals with experimental results and the effectiveness of the proposed methods. Finally the conclusion is given in Section 5 .

\section{Hardware plane of banknote classification}

The banknote image is acquired by a Contact Image Sensor(CIS), when banknote runs in the channel at speed $150 \mathrm{~m} / \mathrm{s}$. The CIS is a kind of line array sensor. One line gray value on face of the object is got while once exposure is executed by CIS. Many lines compose of a frame image. The exposure and data store of CIS are controlled by a Xilinx XC3S200 FPGA, in which a synchronous timing logic circuit is implemented. The invariant feature extraction and banknote classification are finished in $\mathrm{TI}$ TMS320C6713B DSP. The horizontal and vertical resolution of the image are 100 DPI(4-pixel pre millimeter) and about 25 DPI(1-pixel pre millimeter), respectively. Figure 1 shows the hardware plane and CIS. It is a typical signal processing hardware architecture. Some other resources are included in this

\footnotetext{
Proceedings of the 11th Joint Conference on Information Sciences (2008)

Published by Atlantis Press (c) the authors
} 
circuit, such as SDRAM, in order to expand the functions of the classifier, e.g. multi-spectrum fake verification, serial number recognition.

The movement of banknote in channel is complex. There is horizontal movement besides vertical movement, because the speed of left and right side is different when banknote is dragged in channel. As shown in Figure 2, the banknote image is distorted.

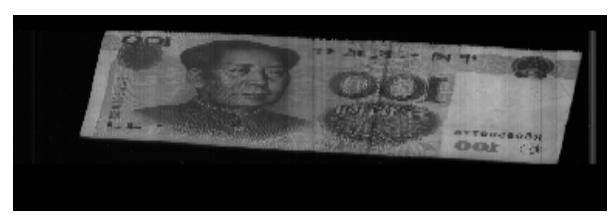

Figure 2. A banknote image acquired by CIS

\section{Invariant Feature Extraction}

\subsection{Variant of scale, rotation and translation}

In mechanical equipment, the belt drives the banknote pass the CIS. Because of slippage, the speed of belt and banknote is different. Usually, the speed of banknote is less than the speed of belt. Assuming the speed of belt is $V_{0}$, and speed of banknote is $V_{1}$, then $V_{0}=V_{1}+v, v \geq 0$. Even the speed of belt is tracked by FPGA in hardware plane, the movement of belt can't reflect the movement of banknote. Therefore, the height of banknote image is various. In other word, the scale of object in banknote is various. Because the speed of left and right belt is also different, the rotation is to occur. In our banknote sorter, the maximum rotation degree is about $\pm 15^{\circ}$. In real case, it is clear that translation is to occur. Variants of scale, rotation and translation bring difficulties for feature extraction and banknote classification. It is necessary to extract the stable features, which are not sensitive to variants of scale, rotation and translation.

\subsection{Feature vector}

In this paper, the object in banknote image is segmented into $M \times N$ blocks, as shown in Figure 3. Considering the real-time requirement, the average gray value in each block is one element of feature vector. Average gray value is the first order statistic, whose computation complexity is very small. Let $F$ be the feature vector, and normalized $f_{i, j}$ be the feature element in the $i$-th row and the $j$-th collum:

$$
F=\left[f_{0,0}, \cdots, f_{0, N-1}, f_{1,0}, \cdots, f_{1, N-1}, \cdots, f_{M-1,0}, \cdots, f_{M-1, N-1}\right]^{T}
$$




$$
\begin{gathered}
\bar{f}_{i, j}=\frac{1}{P \times Q} \sum_{p=0}^{P-1} \sum_{q=0}^{Q-1} v(p, q) \\
f_{i, j}=\bar{f}_{i, j} / \sum_{i=0}^{M-1} \sum_{j=0}^{N-1} \bar{f}_{i, j}
\end{gathered}
$$

where $v(p, q)$ is the gray value at location $(p, q)$ in the block $(i, j)$.

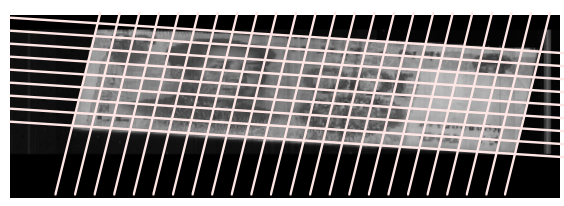

Figure 3. The blocks in banknote image

\subsection{Location of feature block}

Assume that the width and height of banknote image are $W$ and $H$, respectively. From the point $(W / 2,0)$

to $(W / 2, H / 2)$, along the vertical direction, the first pixel $p_{t, 0}$ which is on the top edge of banknote, is found. Its gray value is clearly greater than the one of background. The coordinate of this pixel is recorded as $\left(w_{0}, h_{0}\right)$, where $w_{0}=W / 2$. Then the searching range is moved to from $\left(w_{0}-s, h_{0}-\varepsilon\right)$ to $\left(w_{0}-s, h_{0}+\varepsilon\right)$, where $s$ is the step size, and $\varepsilon$ is the vertical range. In this range, the second pixel $p_{t,-1}$ on the top edge of banknote is found. The coordinate of $p_{t,-1}$ is recorded as $\left(w_{-1}, h_{-1}\right), \quad$ where $\quad w_{-1}=w_{0}-s$, $h_{0}-\varepsilon \leq h_{-1} \leq h_{0}+\varepsilon$. By the same means, the next pixel $p_{t,-2}$ is found, whose coordinate is $\left(w_{-2}, h_{-2}\right)$, where $w_{-2}=w_{-1}-s$

$h_{-1}-\varepsilon \leq h_{-2} \leq h_{-1}+\varepsilon$. If the searching range is moved to the right side of the first pixel $p_{t, 0}$, the other serial of pixels will be found on the top edge of banknote. All of the pixels compose of a point set $P_{t}$ as follows:

$P_{t}=\left\{p_{t,-R}, p_{t,-R+1}, \cdots, p_{t,-1}, p_{t, 0}, p_{t, 1}, \cdots, p_{t, S-1}, p_{t, S}\right\}$

Each point $p_{t, i}$ in this set is on the top edge of banknote. The middle point $p_{t, 0}$ 
is always found at first. The next point is searched in the range that is assigned based on the coordinate of the previous point and parameters $S$ and $\varepsilon$ by the use the following expression:

$$
\begin{aligned}
& w_{i}=w_{i-\operatorname{sgn}(i)}+\operatorname{sgn}(i) s, i \neq 0 \\
& h_{i} \in\left\{\begin{array}{l}
h \mid h \in Z, \\
\min \left(0, h_{i-\operatorname{sgn}(i)}-\varepsilon\right) \leq h \leq \max \left(h_{i-\operatorname{sgn}(i)}+\varepsilon, H / 2\right), i \neq 0
\end{array}\right\}
\end{aligned}
$$

The number of points in the set $P_{t}$ is $R+S+1$. If the value of $R+S+1$ is less than a threshold value, e.g. 20 , or the first point $p_{t, 0}$ can't be found, it means that the banknote is destroyed seriously. Then the banknote will be rejected. By the same method, the point sets of bottom, left, and right edges will be structured.

The Bayesian MAP method is used to fit the edge linear equation ${ }^{[5]}$. The top edge linear equation is defined as $L_{t}: y=k_{t} x+b_{t}$. Similarly, the bottom, left and right edges linear equations are $L_{b}: y=k_{b} x+b_{b}$, $L_{l}: y=k_{l} x+b_{l}$ and $L_{r}: y=k_{r} x+b_{r}$, respectively. The parameters $k_{\bullet}$ and $b$. are estimated by linear equation:

$$
\left(\begin{array}{cc}
R+S+1 & \sum_{i} w_{i} \\
\sum_{i} w_{i} & \sum_{i} w_{i}^{2}
\end{array}\right)\left(\begin{array}{l}
k_{\bullet} \\
b_{\bullet}
\end{array}\right)=\left(\begin{array}{c}
\sum_{i} h_{i} \\
\sum_{i} h_{i} w_{i}
\end{array}\right)
$$

The angles of inclination in horizontal and vertical directions are computed as:

$$
\theta_{h}=\left[\arg \tan \left(k_{t}\right)+\arg \tan \left(k_{b}\right)\right] / 2
$$

$\theta_{v}=\left\{\begin{array}{cc}\pi / 2 & \text { if } k_{l} \rightarrow \infty \text { or } k_{r} \rightarrow \infty \\ {\left[\arg \tan \left(k_{l}\right)+\arg \tan \left(k_{r}\right)\right] / 2} & \text { otherwise }\end{array}\right.$

The four coordinates of points of intersection can be get through solving the linear equations $L_{t}, L_{b}, L_{l}$, and $L_{r}$. They are left-top point $\left(x_{0}, y_{0}\right)$, left-bottom $\left(x_{1}, y_{1}\right), \quad$ right-bottom $\left(x_{2}, y_{2}\right)$, and right-top $\left(x_{3}, y_{3}\right)$. The width of banknote $W_{b}$ in an image is the distance between lines $L_{l}$ and $L_{r}$. And the height of banknote $H_{b}$ is the distance between lines $L_{t}$ and $L_{b}$. The $W_{b}$ and $H_{b}$ are computed by: 


$$
\begin{aligned}
& W_{b}=\frac{1}{2}\left[\sqrt{\left(x_{0}-x_{3}\right)^{2}+\left(y_{0}-y_{3}\right)^{2}}+\sqrt{\left(x_{1}-x_{2}\right)^{2}+\left(y_{1}-y_{2}\right)^{2}}\right] \\
& (10) \\
& H_{b}=\left(k_{b} x_{0}-y_{0}+y_{1}\right) / \sqrt{k_{b}^{2}+1}
\end{aligned} \quad \Delta j=\left\{\begin{array}{cc}
0 & \text { if } \theta_{v}=\pi / 2 \\
2 j /\left(k_{l}+k_{r}\right) & \text { otherwise }
\end{array}\right.
$$

Then the width and height of a feature block are $W_{b} / M$ and $H_{b} / N$, respectively. Then the linear equations of segmenting lines in Figure 3 can be obtained by the angles of inclination and offset value $W_{b} / M$ and $H_{b} / N$.

The width and height of object in banknote image are adaptively various based on the scale of banknote. And they are independent of the location of object in banknote image. Therefore, the feature that is extracted from the block is invariant of scale and translation.

Let the coordinate of a pixel in a feature block be $(i, j)$, $i \in\left\{0,1, \cdots, H_{b}\right\}, j \in\left\{0,1, \cdots, W_{b}\right\}$.

Due to the rotation of banknote in image, it is necessary to compute the offset values in both horizontal and vertical direction. Because the size of feature block is small, these offset values can be approximately computed by the following equations:

Then the active coordinate of a pixel in a

feature is $(i+\Delta i, j+\Delta j)$.

In (12) and (13), the offset values are computed based on the angles of inclination. Therefore, the coordinates of pixels in each feature block is not sensitive to the rotation. The average feature element that is extracted by (2) and (3) in a feature block is invariant of rotation.

\section{Experimental results}

In this experiment, the banknote images of the Chinese RMB, euro, and U.S.dollar are acquitted by hardware plane and CIS. The 3-layer BP neural networks are used to classify these images.

In feature extraction, the step size of horizontal and vertical edge detection $S$ is set to 6 and 4 , respectively. The vertical searching range $\varepsilon$ is set to 8 . The number of feature blocks in horizontal, $M$, is set to 8 , and the number of feature blocks in vertical direction, $N$, is various. The number of 
Figure 4. The relationship between

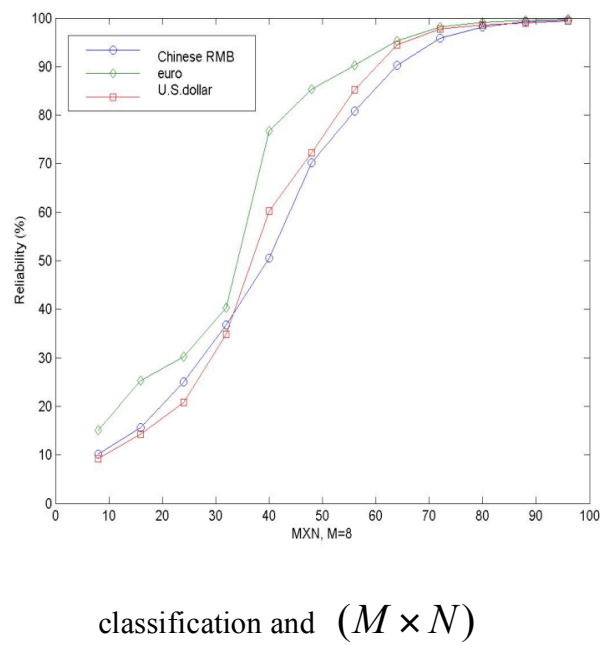

feature elements is $(M \times N)$. The relationship of classification reliability and $(M \times N)$ is shown in Figure 4 .

Table 1 shows that the classification correct rate of different banknotes. In this table, the face value and face direction of euro and U.S.dollar are not listed.

\section{Conclusion}

In this paper, an invariant feature extraction method has been proposed. This method is insensitive to the variant of scale, rotation and translation. The computation complexity of the feature extraction is low, ant it can meet to the requirement of real-time. The experimental results show that the method is effective to decrease the data variant and improve the reliability of banknote classification.

\section{References}

[1] A. Frosono, M. Cori and P. Priami. A Neural Network-Based Model for Paper Currency Recognition and Verification. IEEE Transactions on Neual Network . 1996, 2(6). $1428-1490$

[2] Takeda, F. and Omatu, S. High speed paper currency recognition by neural networks. IEEE Transaction on Neural Networks, 1995, 16(1), 73-77

[3] A. Ahmadi, S. Omatu and T. Fujimaka. Improvement of Reliability in Banknote Classification Using Reject Option and Local PCA. Information Sciences. 2004(168), 277-293

[4] A. Verikas, K. Malmqvist and L. Bergman. Detecting and Measuring Rings in Banknote Images. Engineering Application of Artificial Intelligence. 2005(18), 363-371

[5] P. C. Gregory. Bayesian Logical Data Analysis for the Physical Sciences: A Comparative Approach with Mathmatica Support. Cambridge University Press. Cambridge, UK. $243-283$

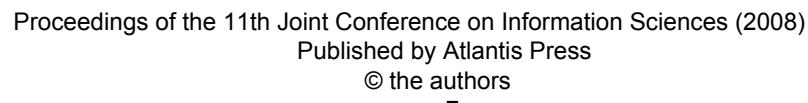


Table 1. The classification correct rate with parameters

$$
M=8, N=96,(\text { horizontal }) s=6,(\text { vertical }) s=4, \varepsilon=8
$$

\begin{tabular}{|c|c|c|c|c|c|}
\hline Currency & $\begin{array}{l}\text { Face } \\
\text { value }\end{array}$ & Face direction & $\begin{array}{l}\text { No. of Training } \\
\text { samples }\end{array}$ & $\begin{array}{l}\text { No. of testing } \\
\text { samples }\end{array}$ & $\begin{array}{l}\text { Correct } \\
\text { rate }(\%)\end{array}$ \\
\hline \multirow{20}{*}{ Chinese RMB } & \multirow{4}{*}{5} & Front-Up & 200 & \multirow{20}{*}{10000} & 99.95 \\
\hline & & Front-Down & 200 & & 99.90 \\
\hline & & Reverse-Up & 190 & & 99.99 \\
\hline & & Reverse-Down & 250 & & 100.00 \\
\hline & \multirow{4}{*}{10} & Front-Up & 197 & & 99.90 \\
\hline & & Front-Down & 201 & & 99.92 \\
\hline & & Reverse-Up & 210 & & 100.00 \\
\hline & & Reverse-Down & 211 & & 100.00 \\
\hline & \multirow{4}{*}{20} & Front-Up & 200 & & 99.95 \\
\hline & & Front-Down & 200 & & 99.97 \\
\hline & & Reverse-Up & 199 & & 100.00 \\
\hline & & Reverse-Down & 206 & & 100.00 \\
\hline & \multirow{4}{*}{50} & Front-Up & 205 & & 99.99 \\
\hline & & Front-Down & 200 & & 100.00 \\
\hline & & Reverse-Up & 200 & & 100.00 \\
\hline & & Reverse-Down & 200 & & 100.00 \\
\hline & \multirow{4}{*}{100} & Front-Up & 200 & & 100.00 \\
\hline & & Front-Down & 243 & & 100.00 \\
\hline & & Reverse-Up & 189 & & 100.00 \\
\hline & & Reverse-Down & 192 & & 100.00 \\
\hline euro & -- & -- & 4300 & 25000 & 99.99 \\
\hline U.S.dollar & -- & -- & 4000 & 25000 & 99.98 \\
\hline
\end{tabular}

